\title{
PHOTOACOUSTIC EFFECT IN MICRO- AND NANOSTRUCTURES: NUMERICAL SIMULATIONS OF LAGRANGE EQUATIONS
}

O. Romanov, Ya. Shtykov, I. Timoshchenko

Belarusian State University, Minsk, Belarus

romanov@bsu.by

\section{INTRODUCTION}

The photoacoustic effect is the formation of sound waves due to absorption of the modulated light pulse by irradiated medium. At the present time, the photoacoustic effect is widely used in biomedical studies, photoacoustic spectroscopy etc. Of particular interest are studies of the interaction of pulsed laser radiation with absorbing micro- and nanostructures. Absorption of laser pulse energy in micro- and nanostructures allows exciting acoustic oscillations in the frequency range from gigahertz to terahertz. Terahertz frequency acoustic vibrations are of special interest for fundamental research and have numerous potential applications (acoustic imaging of nano-objects, acoustic nanocavities, phononic crystals).

\section{METHOD AND RESULTS}

This work provides the description of theoretical and numerical modeling techniques of thermomechanical effects that take place in absorbing one-, two- and three-dimensional micro- and nanostructures of different materials under action of pulsed laser radiation. A proposed technique of the numerical simulation is based on the solution of equations of motion of continuous media in the form of Lagrange for a spatially inhomogeneous media. This model allows calculating fields of temperature, pressure, density and velocity of the medium depending on the parameters of laser pulses and the characteristics micro- and nanostructures. The problems of excitation and propagation of acoustic oscillations in homogeneous and heterogeneous media and structures are considered, and the potential applications of the photoacoustic effect in medicine and nanotechnology are discussed. Examples of implementation of the model in connection with possible applications in the area of nanophotonics, nanoacoustics, and nanoplasmonics are presented.

Absorption of the energy of a pulsed laser beam in the medium leads to its local heating, thermal expansion and generation of acoustic (shock) waves. These processes can be described by the equations of motion of the medium in the form of Lagrange. For many practical cases, it is informative modeling of one-dimensional tasks (planar, cylindrical or spherical) for appropriate geometry of energy release [1]. Based on this approach, the processes of excitation of acoustic waves in one-dimensional metallic [2] and carbon [3] nanostructures (nanofilms, nanowires, spherical micro- and nanoparticles) have been investigated. The heating of metals under the action of ultrashort laser pulses is described in terms of two-temperature model 
for the electron gas and the ionic lattice, and spatial-temporal dynamics of excitation and propagation of terahertz acoustic oscillations is investigated based on the numerical solution of equations of motion of layered media, and two-dimensional plasmonic nanostructures [4, 5].

\section{CONCLUSIONS}

As a generalization, for theoretical description of thermomechanical phenomena in three-dimensional absorbent nanostructures under the action of pulsed laser radiation a technique has been developed for numerical simulation of three-dimensional equations of motion of continuous media in the form of Lagrange and three-dimensional heat conduction equation. The fields of temperature, pressure, density and velocity of the medium have been calculated for different geometries of micro- and nanostructures.

\section{REFERENCES}

[1] O. Romanov, G. Zheltov, G. Romanov, "Numerical modeling of thermomechanical processes in absorption of laser radiation in spatially inhomogeneous media", Journal of Engineering Physics and Thermophysics, Vol. 84, Issue 4, pp.772-780, 2011.

[2] O. Romanov, G. Zheltov, G. Romanov, "Action of Femtosecond Laser Pulses on Metal Nanoparticles in a Liquid", Bull. of the Rus. Acad. of Sc.: Phys., Vol. 75, Issue 12, pp.1589-1591, 2011.

[3] L. Golubewa, I. Timoshchenko, O. Romanov, R. Karpicz, T. Kulahava, D. Rutkauskas, M. Shuba, A. Dementjev, Yu. Svirko, P. Kuzhir, "Single-walled carbon nanotubes as a photo-thermo-acoustic cancer theranostic agent: theory and proof of the concept experiment", Scientific Reports, Vol. 10, pp.22174, 2020.

[4] N. Khokhlov, G. Knyazev, B. Glavin, Y. Shtykov, O. Romanov, V. Belotelov, "Interaction of surface plasmon polaritons and acoustic waves inside an acoustic cavity", Opt. Lett., Vol. 42, pp. 3558-3561, 2017.

[5] V. Belotelov, A. Kalish, G. Knyazev, E. Nguen, O. Romanov, A. Tolstik, "Optoacoustical transducer based on plasmonic nanoparticles", Nonl. Phen. in Compl. Syst., Vol. 22, Issue 1, P.55-63, 2019. 